\title{
Balloon dilatation of the aortic valve in adults: a physician's view
}

\author{
K J Beatt
}

The treatment of symptomatic aortic stenosis in elderly patients by percutaneous balloon dilatation was introduced by Cribier in 1985 although the procedure had already been used successfully in children with stenosis of the pulmonary and aortic valves. Until 1985 the only effective treatment was surgery-aortic valvotomy or aortic valve replacement. In the elderly, aortic valve replacement is associated with considerable morbidity and perioperative mortality ranging from $3 \%^{1}$ to $18 \%^{2}$ with even higher rates $(23 \%)$ in patients who are more than 75 years old. ${ }^{3}$ Most of the reported series were completed some time ago and may not reflect current surgical practice. But patients in any surgical series have been selected because they are a good risk; whereas balloon dilatation of the aortic valve is generally performed in patients who are regarded as high risk candidates for surgery or who have been refused surgical treatment. The main purpose of invasive treatment is to relieve symptoms; one year survival of untreated elderly patients is as low as $57 \%$, and even after successful valve replacement or successful balloon dilatation survival cannot be expected to be greatly improved.

Percutaneous balloon dilatation of the aortic valve is currently performed by various related techniques that have become increasingly refined. The technique is simple to perform but as with other interventional procedures the results and complication rates reflect the experience of the operator. This point must be taken into account in interpreting some of the reported series and in assessing the value of the technique. For example in two of the larger series of patients treated by balloon dilatation, one from Europe ${ }^{5}$ and the other from the United States, ${ }^{6}$ mean aortic valve areas increased from 0.49 to $0.93 \mathrm{~cm}^{2}$ and from 0.6 to $0.9 \mathrm{~cm}^{2}$, with $41 \%$ and $19 \%$ of patients having an aortic valve area greater than $1 \mathrm{~cm}^{2}$, a value widely associated with a good chance of a favourable medium term outcome. In contrast some series report valve increases as small as from 0.41 to $0.60 \mathrm{~cm}^{2}$, which cannot be expected to produce sustained haemodynamic improvement. These small increases may be related to the use of a single, possibly undersized, balloon.

Reliable assessment of the results is hampered by the lack of a sufficiently precise method of assessment. Echocardiography including Doppler assessment is not sufficiently objective and variations in both the pressure gradient and the calculated valve area are exaggerated by the large changes in flow and filling pressures that occur during the procedure. We need a better method of distinguishing between a poor result and restenosis of the valve.

The mechanism by which dilatation achieves the improvement is not well understood. In elderly patients aortic stenosis is usually caused by degenerative changes. The valve leaflets are rigid, often with extensive calcification. In general commissural fusion is not an important factor. Therefore the commissural splitting produced by balloon dilatation, which is known to be important in rheumatic aortic stenosis, is of little relevance in calcific aortic stenosis. The most commonly accepted explanation for the improvement after balloon dilatation, which is based on limited experimental evidence, is that a combination of stretching of the aortic valve ring and fracture of the calcified "skeleton" of the valve increases the mobility of the valve leaflets. In the absence of any more dramatic mechanism of action it is perhaps not surprising that only relatively modest increases in valve area are recorded. None the less, because of the curvilinear relation between aortic valve area and valve gradient small increases in valve area produce important haemodynamic and functional improvement.

Balloon dilatation of the aortic valve is associated with a mortality of about $1 \%$ and an in-hospital mortality of $<5 \%$ (most deaths are caused by progressive heart failure). Minor complications of gaining arterial access at the femoral puncture site are common and because they may lead to a general anaesthetic being required in frail elderly patients their effects on outcome should not be underestimated. Complications-such as stroke, myocardial infarction, tamponade, and aortic regurgitation-are less frequent but not insignificant. If the procedure were curative the complication rate would be acceptable, but long term follow up showed that the short term benefits are not well maintained. A study by Commeau et al found that at repeat cardiac catheterisation eight days after dilatation most of the initial benefit had been lost though there was still significant improvement over values before dilatation. ${ }^{7}$ Most studies show that at six months only $50 \%$ of patients continue to benefit from the procedure and in 
selected patients the mortality at one year may be no different from that in untreated patients. ${ }^{8}$ However, comparisons of this type, of the total population with high risk subgroups, may give rise to misleading conclusions and this comment is relevant to all comparisons of operations of the aortic valve. Most of our patients with recurrence of symptoms were operated on and, despite initial reservations by the surgeons, all have done well. ${ }^{9}$ There is little doubt that the introduction of percutaneous balloon dilatation of the aortic valve has stimulated the surgeons to review their indications for aortic valve replacement in the elderly, and that this has resulted in a less conservative approach to these patients in many centres.

The technique of percutaneous balloon dilatation of the aortic valve is still being developed. In children or in patients with small aortic roots it has been shown to be of value. The group of patients who are likely to benefit most from this procedure are the thousands of younger patients in developing countries who die each year of untreated rheumatic aortic stenosis. Most of these patients do not have the opportunity to have surgical treatment and this is unlikely to change soon. The use of imaging techniques such as intravascular ultrasound, which is capable of producing high resolution images of valve leaflets, may allow more effective non-surgical treatment. The ability to perform selective commissurotomy and immediately to assess both the functional result and the anatomical result will lead to renewed interest in balloon dilatation.
Currently balloon dilatation can be expected to produce immediate symptomatic relief with acceptable complication rates in most patients. Long term success is low in the elderly and the technique cannot be considered as an alternative in those who are suitable candidates for surgery. Appropriate patient selection is crucial and the procedure is perhaps most useful in providing a "bridge" to surgery. It should be performed in a limited number of centres by experienced operators who are able to maintain their expertise by operating regularly.

1 Hochberg MS, Morrow AG, Michaelis LL, McIntosh CL, Redwood DR, Epstein SE. Aortic valve replacement in the elderly. Arch Surg 1977;112:1475-80.

2 Quinlan R, Cohn LH, Collins JJ Jr. Determinants of survival following cardiac operations in elderly patients. Chest 1975;68:498-500.

3 Cabrol C, Gandjbakhch I, Pavie A. La chirurgie de remplacement valvulaires acquises. Paris: Flammarion Medecine-Sciences, 1985:513-46.

4 O'Keefe JH Jr, Vliestra RE, Bailey KR, Holmes DR Jr. Natural history of candidates for balloon aortic valvuloplasty. Mayo Clin Proc 1987;62:986-91.

5 Cribier A, Savin T, Berland J, et al. Percutaneous transluminal balloon valvuloplasty of adult aortic stenosis: report of 92 cases. J Am Coll Cardiol 1987;9:381-6.

6 Safian RD, Berman AD, Diver DJ, et al. Balloon aortic valvuloplasty in 170 consecutive patients. $N$ Engl J Med valvuloplasty in 170

7 Commeau P, Grollier G, Lamy E, et al. Percutaneous balloon dilatation of calcific aortic valve stenosis: balloon dilatation of calcific aortic valve stenosis: 1988;59:227-38.

8 Sprigings DC, Jackson G, Chambers JB, et al. Balloon dilatation of the aortic valve for inoperable aortic stenosis. Br Med J 1988;297:1007-10.

9 Serruys PW, Luijten HE, Beatt KJ, et al. Percutaneous balloon valvuloplasty for calcific aortic stenosis. A treatment "sine cure". Eur Heart J 1988;9:782-94. 